\title{
Performance Evaluations of Image Denoising System based on Nonlinear Diffusion Method in Wavelet Domain
}

\author{
Devendra Moraniya
}

\author{
Mayank Mehra
}

Dhiiraj Nitnawwre, Phd

\author{
IET DAVV Indore, India
}

\begin{abstract}
Image processing is always being a research field for the researcher. The Image denoising is one of the important areas of image processing. There are several methods for image denoising in spatial and transform domain. The current trends of the image denoising research are the evolution of mixed domain methods. In this paper, a mixed domain image denoising method is proposed, which is based on the wavelet transform, median filter and nonlinear diffusion. The wavelet transform is used in this paper to convert the spatial domain image to wavelet domain coefficients. The detail component are removed due to the most of the image part is in approximation part. The approximation coefficient is then filtering by nonlinear diffusion and median filter separately. The peak signal to noise ratio (PSNR), root mean square error (RMSE) and mean structural similarity index matrix (MSSIM) are used as the performance parameter. The different wavelet families are used to optimize the performance of denoising. The Coiflet 2 wavelet and diffusion algorithm are giving the best denoising result.
\end{abstract}

\section{Keywords}

Wavelet Transform, Perona and Malik (PM2), PSNR, RMSE, MSSIM etc.

\section{INTRODUCTION}

A digital image is generally represented as a matrix of grey level or color values. Blur is the attenuation of high frequencies may result in smooth edges in the image. A good quality image has about 256 grey values, where 0 represents black and 255 represents white. Images are often corrupted by noise during the, processing, compression, transmission, and reproduction which reduces the image quality. A common problem with image is the contamination due to undesired Noise. Image denoising is the method of estimating the noise free image from the noisy observation. In the process of noise removal many denoising methods consider noise as high frequency content, and consider the edges with noise and Smoothing. An edge contains structural information that should be preserved. Nonlinear diffusion, initially proposed by Perona Malik in 1990, is a popular iterative method for noise removal that uses to perform edge preserving smoothing [1].There are many methods for image denoising. Simple mathematical filters such as average filter, median filter Gaussian filter, wavelet transform, soft thresholding, and anisotropic diffusion, are some of the techniques applied for image denoising [2]. Wavelet analysis is one of the powerful methods to reduce image noise. In this work we developed new concept, wavelet family is used for image denoising and to denoise image which is corrupted by Gaussian noise. The intention behind this method is to improve result than the Haar wavelet transforms [3].The proposed method produces improved results. Wavelet is a mathematical tool that can be used to retrieve information from images. Wavelet $\psi$ is a function of 0 averages, having the energy concentrated in time and more flexible extracting time and frequencies information a family of wavelet can be constructed from a function $\psi(\mathrm{t})$ ,also known as mother wavelet. The procedure for noise reduction is applied on the wavelet coefficients obtained after applying the wavelet transform to the image at different scales .wavelet transform produces approximation, horizontal detail, vertical detail and diagonal detail coefficient which represent the various spatial frequency bands. The wavelet transform is used because it is good for energy compaction since the small and large coefficients are more likely due to noise and important image feature. And the diffusion algorithm is providing higher speed in such image region where the magnitude gradient is small vice versa. Our concept we use Gaussian noise \& wavelet family. Perona and Malik (PM) who proposed a non linear diffusion process, where diffusion can take place with a variable diffusion in order to control the smoothing effect. The diffusion coefficient in the PM2 process was chosen to be a decreasing function of the gradient of the signal. This operation selectively low passes filter regions that do not contain large gradient [4] [5].

This paper is organized as follows. Firstly this paper presents the Image denoising technique in section 2. Section 3 describes the proposed algorithm. Section 4 shows the experimental results and conclusion is given in section 5 .

\section{IMAGE DENOISING TECHNIQUE}

\subsection{Non linear diffusion based image denoising}

The theory of nonlinear diffusion is using nonlinear partial differential equation (PDEs).This method is used to create a scale space representation that contains simplified images. The image edges of some images are maintained or enhanced. It is expected that the large values of diffusivity for the small gradient norm for performing strong smoothing, and for low values of diffusivity for the high gradient norm to slow down the diffusion process and protect delicate image features. To form nonlinear diffusion, it is replaced constant diffusion coefficient of linear equation by using a smooth non increasing diffusivity function $g$ with $g(0)=1, g(s) \geq 0$, and $\lim \mathrm{s} \longrightarrow \infty \mathrm{g}(\mathrm{s})=0$. As a result, the diffusivities become variable in both space and time. The Perona-Malik equation is [1].

$$
\frac{\delta u}{\delta t}=\nabla \cdot(g(|\nabla u|) \nabla u)
$$


With homogeneous boundary conditions and the initial condition $u^{0}(x)=\mathrm{f}(x)$, f denoting the input image. Perona and Malik suggest the diffusivity function:

$$
g(s)=1 /\left(1+s^{2} / \lambda^{2}\right),
$$

Where $\lambda$ indicates contrast parameter.

Here below the Perona \& Malik equation is shown in 1D which demonstrates the role of contrast parameter $\lambda[6]$.

$$
\frac{\delta u}{\delta t}=\frac{\delta}{\delta x}\left(g\left(\left|u_{x}\right|\right) u_{x}\right)=\Phi^{\prime}\left(u_{x}\right) u_{x x}
$$

With $g\left(\left|u_{x}\right|\right)=1 /\left(1+\left|u_{x}\right|^{2} / \lambda^{2}\right) \quad$ Here $\delta$ t subscripts denote partial derivatives, and the diffusion time is a simplification parameter and diffusion time is large values corresponding to stronger filtering.

$g\left(\mid u_{x}\right)$ is non negative function that controls the amount of diffusion.

The desirable characteristics of diffusion coefficient are such that:

1. It diffuses more in smooth areas and less around large intensity transitions.

2. Around the large intensity transitions it faces backward diffusion so that edges are sharpened and assure forward diffusion in smooth areas for noise removal.

\subsection{Discrete wavelet transforms}

Signal is represents as a superposition of two sorts of element: scaling functions and wavelets. The scaling functions represents the signal with a lower resolution approximation (low pass filter) while the wavelets represents the resulting difference components (high pass filters). A representation is the so called discrete wavelet transform

$$
\mathrm{f}(\mathrm{t})=\sum \mathrm{c}(\mathrm{k}) \varphi_{\mathrm{k}}(\mathrm{t})+\sum \sum \mathrm{d}(\mathrm{j}, \mathrm{k}) \psi_{\mathrm{j},}(\mathrm{t})
$$

Where $\varphi(t)$ is the scaling function, $\psi(t)$ is the wavelet. The data is separated in to different frequency components by this tool and after that it studies each component with resolution matched to its scale. DWT is computed with a cascade of filtering followed by a factor 2 sub sampling [7].

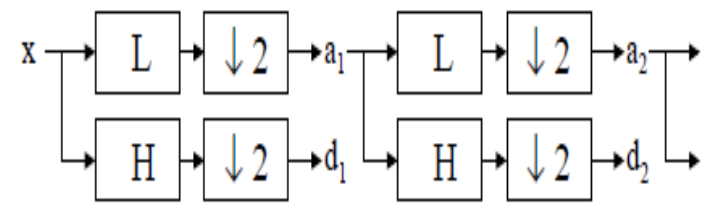

$\mathrm{H}$ and $\mathrm{L}$ denote high and low pass filters respectively. $\downarrow 2$ denotes sub sampling. Outputs of the filters are represents by equations 1 and 2[7].

$$
\begin{gathered}
\mathrm{a}_{\mathrm{j}+1}[\mathrm{p}]=\sum_{n=-\infty}^{+\infty} \mathrm{l}[\mathrm{n}-2 \mathrm{p}] \text { aj }[\mathrm{n}] \\
\mathrm{d}_{\mathrm{j}+1}[\mathrm{p}]=\sum_{n=-\infty}^{+\infty} \mathrm{h}[\mathrm{n}-2 \mathrm{p}] \text { aj }[\mathrm{n}]
\end{gathered}
$$

Where aj is used to next scale of the transform and element $\mathrm{dj}$ is wavelet coefficients. $1[n]$ and $h[n]$ are coefficients of low pass and high pass filters.

\subsubsection{Haar wavelet}

Haar wavelet is non continuous function and resembles a step function. Haar wavelet \& scaling functions are given respectively by

$$
\Psi(\mathrm{x})=1_{[0,1 / 2]}-1_{[1 / 2,1]}
$$

$$
\Phi(\mathrm{x})=1_{[0,1]}
$$

Where $1[a, b)$ denotes the characteristics function equal to 1 on [a, b) and zero everywhere else [8] [9].

(i) It is real and orthogonal and compact support. Therefore

$$
\begin{aligned}
& \mathrm{Hr}=\mathrm{Hr}^{*} \\
& \mathrm{Hr}^{1}=\mathrm{Hr}^{\mathrm{t}}
\end{aligned}
$$

And have the following advantage

(1)Computation time is the best in performance.

(2)Simplicity.

(3) With high computation of speed.

\subsubsection{Daubechies and Coiflets}

Daubechies (db) and Coiflet (coif) wavelets are families of orthogonal wavelets that are compactly supported. Compactly supported wavelets correspond to finite-impulse response (FIR) filters and, thus, lead to efficient implementation [10].The particular class of wavelets known as coiflets is near symmetric. They have a same number of vanishing moments for the scaling function and wavelets. The wavelet is near symmetric their wavelet functions have N/3 vanishing moments and scaling functions N/3-1 .In addition coiflets have been shown to be excellent for the sampling approximation of smooth functions. Both the scaling function (low pass filter) the wavelet function (High pass filter) must be normalized by a factor $1 / \sqrt{2}[11]$.

\section{PROPOSED ALGORITHM}

1. Read the image.

2. Add the Gaussian noise to the image.

3. Apply DWT using Harr wavelet, Db2 wavelet and Coiflet wavelet over the mixer of the noise and the Original image.

4. DWT will generate the approximation coefficient and detail coefficient..

5. The approximation coefficients apply non-linear diffusion algorithms. The nonlinear diffusion algorithm apply at low frequency and wavelet transform will use at high frequencies.

6 . Reconstruct an estimate of the original image by applying the corresponding IDWT.

7. It will calculate and display the result of the PSNR, RMSE and MSSIM.

8. Compare all the result of different method at different noise level.

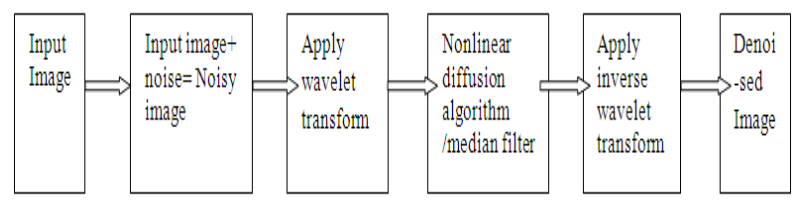

Fig 1: Show the denoising algorithm 


\section{EXPERIMENTAL RESULT}

We have taken standard image Lena $(512 \times 512)$. Add the Gaussian noise to the image. Perform image denoising via wavelet family (haar wavelet, db2 wavelet, and Coiflet 2 wavelet), nonlinear diffusion, and median filter. The result (PSNR, RMSE, MSSIM) of combination different methods at different noise level show in tables. The Coiflet wavelet and diffusion algorithm are giving the best denoising result.

Table1. PSNR values from different Algorithm

\begin{tabular}{|r|r|r|r|r|r|r|}
\hline Noise & Haar & $\begin{array}{l}\text { Haar+ } \\
\text { median }\end{array}$ & $\begin{array}{l}\text { Diffu } \\
\text {-sion }\end{array}$ & $\begin{array}{l}\text { Haar } \\
\text { +Diff }\end{array}$ & $\begin{array}{l}\text { Db2+ } \\
\text { Diff }\end{array}$ & $\begin{array}{l}\text { Coif2+ } \\
\text { Diff }\end{array}$ \\
\hline 0.01 & 24.68 & 26.24 & 27.1 & 27.22 & 27.98 & 28.16 \\
\hline 0.02 & 22.41 & 25.35 & 26.61 & 26.63 & 27.25 & 27.49 \\
\hline 0.03 & 21.05 & 24.74 & 23.89 & 25.89 & 26.44 & 26.61 \\
\hline 0.04 & 20.03 & 24.13 & 21.35 & 24.96 & 25.42 & 25.53 \\
\hline 0.05 & 19.29 & 23.60 & 19.46 & 24.32 & 24.63 & 24.79 \\
\hline 0.06 & 18.65 & 23.22 & 18 & 23.49 & 23.79 & 23.85 \\
\hline 0.07 & 18.2 & 22.70 & 16.94 & 22.76 & 23 & 22.99 \\
\hline 0.08 & 17.66 & 22.30 & 16.01 & 22.13 & 22.32 & 22.38 \\
\hline 0.09 & 17.29 & 22.04 & 15.28 & 21.61 & 21.76 & 21.75 \\
\hline 0.1 & 17.07 & 21.81 & 14.69 & 21.07 & 21.29 & 21.31 \\
\hline
\end{tabular}

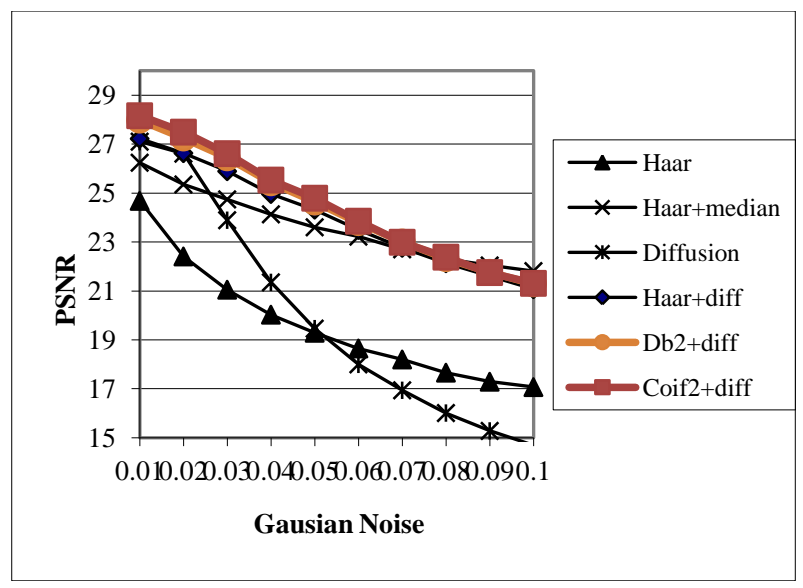

Fig 2: Graph between PSNR Vs Gaussian Noise

Table2. RMSE values from different Algorithm

\begin{tabular}{|l|r|r|r|r|r|r|}
\hline $\begin{array}{l}\text { NOI } \\
\text { SE }\end{array}$ & Haar & $\begin{array}{l}\text { Haar+ } \\
\text { median }\end{array}$ & $\begin{array}{l}\text { Diffu } \\
\text {-sion }\end{array}$ & $\begin{array}{l}\text { Haar } \\
\text { +Diff }\end{array}$ & $\begin{array}{l}\text { Db2 } \\
\text { +Diff }\end{array}$ & \multicolumn{1}{c|}{$\begin{array}{l}\text { Coif2 } \\
\text { +Diff }\end{array}$} \\
\hline 0.01 & 14.87 & 12.41 & 10.02 & 11.09 & 10.09 & 9.09 \\
\hline 0.02 & 19.3 & 13.75 & 11.91 & 11.88 & 11.05 & 10.75 \\
\hline 0.03 & 22.58 & 14.75 & 16.28 & 12.92 & 12.14 & 11.91 \\
\hline 0.04 & 25.38 & 15.84 & 21.81 & 14.16 & 13.52 & 13.4 \\
\hline 0.05 & 27.64 & 16.83 & 27.12 & 15.5 & 14.95 & 14.68 \\
\hline 0.06 & 29.78 & 17.59 & 32.06 & 17.06 & 16.48 & 16.35 \\
\hline 0.07 & 31.35 & 18.68 & 36.24 & 18.54 & 18.04 & 18.06 \\
\hline 0.08 & 33.38 & 19.55 & 40.35 & 19.94 & 19.5 & 19.37 \\
\hline 0.09 & 34.8 & 20.15 & 43.86 & 21.18 & 20.72 & 20.84 \\
\hline 0.1 & 35.69 & 20.68 & 46.96 & 22.53 & 21.97 & 21.92 \\
\hline
\end{tabular}

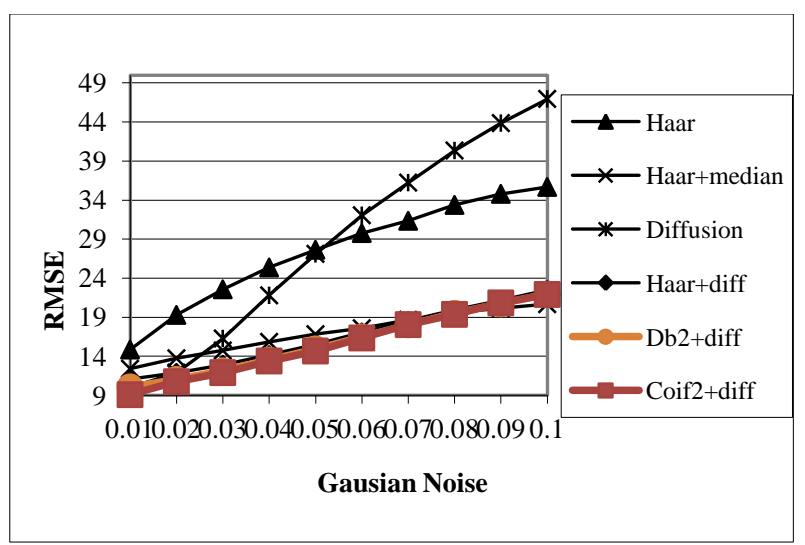

Fig 3: Graph between RMSE Vs Gaussian Noise

Table3. MSSIM values from different Algorithm

\begin{tabular}{|r|r|r|r|r|r|r|}
\hline $\begin{array}{l}\text { NOI } \\
\text {-SE }\end{array}$ & \multicolumn{1}{|c|}{ Haar } & $\begin{array}{l}\text { Haar+ } \\
\text { median }\end{array}$ & $\begin{array}{l}\text { Diffu } \\
\text {-sion }\end{array}$ & $\begin{array}{l}\text { Haar+ } \\
\text { Diff }\end{array}$ & $\begin{array}{l}\text { Db2+ } \\
\text { Diff }\end{array}$ & $\begin{array}{l}\text { Coif2+ } \\
\text { Diff }\end{array}$ \\
\hline 0.01 & 0.51 & 0.69 & 0.76 & 0.76 & 0.78 & 0.78 \\
\hline 0.02 & 0.39 & 0.63 & 0.65 & 0.73 & 0.75 & 0.76 \\
\hline 0.03 & 0.33 & 0.58 & 0.54 & 0.69 & 0.7 & 0.71 \\
\hline 0.04 & 0.29 & 0.55 & 0.46 & 0.63 & 0.65 & 0.66 \\
\hline 0.05 & 0.27 & 0.52 & 0.37 & 0.59 & 0.6 & 0.61 \\
\hline 0.06 & 0.24 & 0.49 & 0.32 & 0.53 & 0.54 & 0.56 \\
\hline 0.07 & 0.22 & 0.48 & 0.28 & 0.49 & 0.51 & 0.52 \\
\hline 0.08 & 0.2 & 0.45 & 0.25 & 0.46 & 0.47 & 0.48 \\
\hline 0.09 & 0.19 & 0.44 & 0.22 & 0.42 & 0.44 & 0.45 \\
\hline 0.1 & 0.18 & 0.43 & 0.2 & 0.4 & 0.42 & 0.42 \\
\hline
\end{tabular}

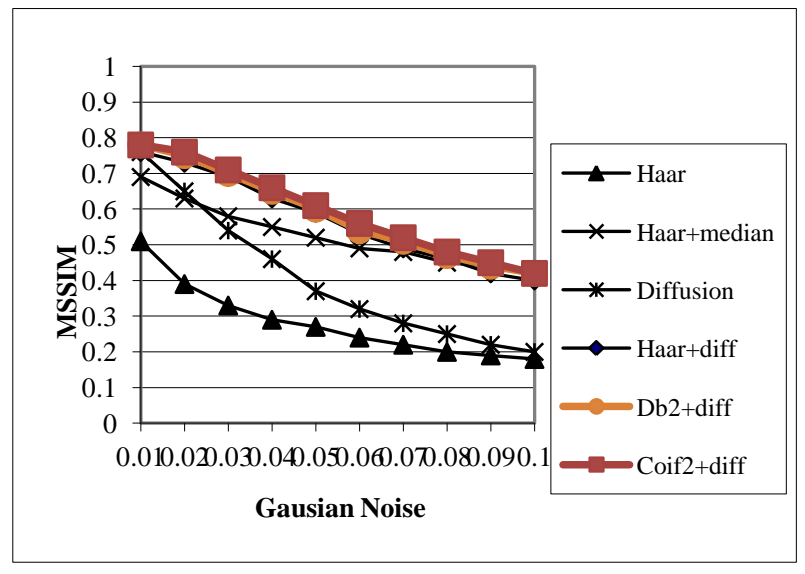

Fig 4: Graph between MSSIM Vs Gaussian Noise 


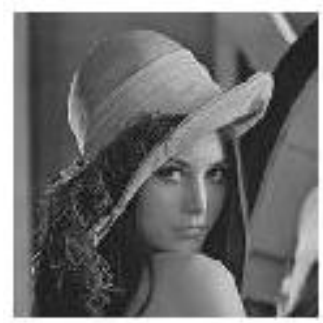

Fig 5: (a)

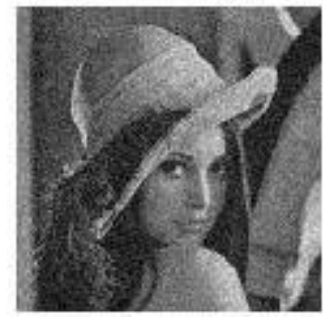

Fig 5: (c)

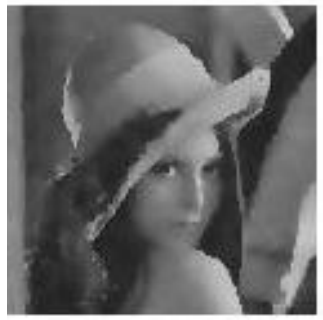

Fig 5: (e)

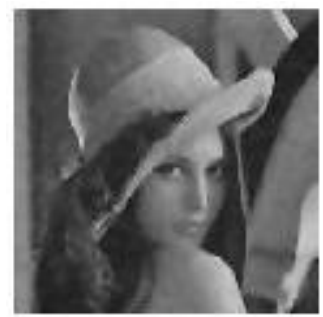

Fig 5: (g)

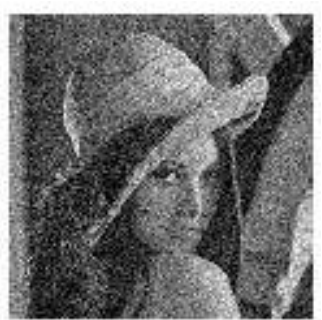

Fig 5: (b)

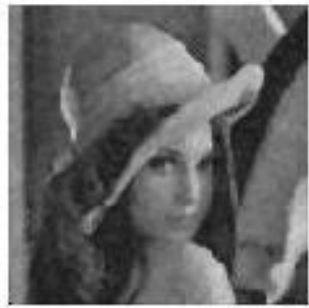

Fig 5: (d)

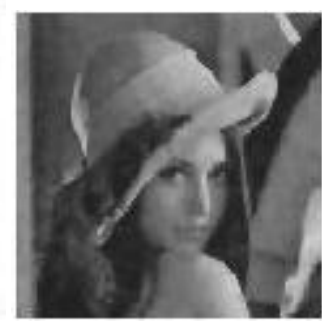

Fig 5: (f)

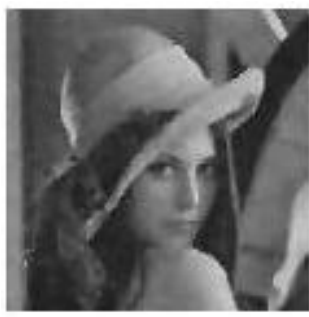

Fig 5: (h)
Fig 5: Images of different algorithm

(a) Original image (b) Noisy image (c) Denoised with haar wavelet (d) Denoised with haar wavelet+ median filter(e) Denoised with nonlinear diffusion algorithm (f) Denoised with haar wavelet+ non linear diffusion algorithm (g) Denoised with $\mathrm{db} 2$ wavelet+ non linear diffusion algorithm (h) Denoised with coiflet2wavelet+ non linear diffusion algorithm .

\section{CONCLUSION}

The denoising performance improved by wavelet family. And improved denoising results achieved by using estimate the wavelet coefficients. Wavelet transforms produce approximation coefficients and detail coefficients. The non wavelet transforms use at higher frequencies coefficients. In linear diffusion applies at low frequencies coefficients.
- From the simulation result we conclude that Coif2+Non linear diffusion algorithm shows better performance in terms of PSNR, RMSE and MSSIM as compare to all other method.

- For future wok - We can train our algorithm using various AI techniques like fuzzy logic or neural network, in order to attain the best output without performing calculations for each and every combination. Use of AI techniques will lead to the optimal solution directly, with more efficiency and less tedious work.

\section{REFERENCES}

[1] P. Perona and J. Malik Scale-space and edge detection using anisotropic diffusion. IEEE Trans on Pattern Analysis and Machine Intelligence, 1990, 12(7):629-639.

[2] J. Babaud, A. Witkin, M. Baudin, and R. Duda, "Uniqueness of the Gaussian kernel for scale-space sfiltering," IEEE Trans. Pattern Anal. Machine Intell, vol. PAMI-8, Jan. 1986.

[3] Yuan Zejian, et al. A design method for nonlinear diffusion filter and its application [J]. Chinese J Computers, 2002, 25(10): 1072-1076

[4] P. Mr'azek, J. Weickert, and G. Steidl.Correspondences between wavelet shrinkage and nonlinear diffusion. In L. D. Griffin and M. Lillholm, editors, Scale-Space Methods in Computer Vision, volume 2695 of Lecture Notes in Computer Science, pages 101-116, Berlin, 2003. Springer.

[5] Forward and Backward Diffusion processes for adaptive image enhancement and denoising IEEE transactions on image processing, vol11, no. 7 July 2002.

[6] J. Weickert. Anisotropic Diffusion in Image Processing.Teubner, Stuttgart, 1998

[7] S. Mallat: A wavelet Tour of Signal Processing, Academic Press, and San Diego 1998.

[8] H. Gao. Wavelet shrinkage denoising using the nonnegative garrote. Journal of Computational and Graphical Statistics, 7(4):469-488, 1998.

[9] H. Gao and A. G. Bruce. Wave Shrink with firm shrinkage. Statistica Sinica, 7:855-874, 1997

[10] I. Daubechies. Ten Lectures on Wavelets. SIAM, Philadelphia, PA, 1992

[11] G. Beylkin, R. Coifman, and V. Rokhlin (1991),Fast wavelet transforms and numerical algorithms, Comm. Pure Appl. Math., 44, pp. 141-183

[12] Sonja Grgic, MislavGrgic, Member, 2001. IEEE and BrankaZovko-Cihlar, Member, IEEE. Performance Analysis of Image Compression Using Wavelets.IEEE Trans., Vol: 48.

[13] P. Raviraj, CSE, Sri Vishnu Engg. College for Women, Bhimavaram, A.P, India, the Modified 2D-Haar Wavelet Transformation in Image Compression Middle-East Journal of Scientific Research 2 (2): 73-78, 2007 ISSN 1990-9233@ IDOSI Publications, 2007. 